\title{
MATERIALIDAD, FORMAS Y CONTENIDOS DEL ESPACIO PÚBLICO EN LÍNEA: LA ELECCIÓN DE GONZALO RAMÍREZ COMENTADA POR LOS USUARIOS DE FACEBOOK
}

\section{MATERIALITY, FORMS AND CONTENT OF THE ONLINE PUBLIC SPACE: THE ELECTION OF GONZALO RAMÍREZ AS COMMENTED BY FACEBOOK USERS}

\author{
Baptiste Kotras*
}

\section{RESUMEN}

Este artículo propone un análisis de los comentarios publicados por los seguidores del sitio CRHoy en Facebook a lo largo de la secuencia política del $1^{\circ}$ de mayo de 2017, en la cual fue electo Gonzalo Ramírez (PRC) como presidente de la Asamblea Legislativa. Busca identificar los códigos y las normas implícitas de la discusión en línea sobre asuntos políticos y describir las principales tendencias en las opiniones espontáneamente elaboradas por las personas comentaristas. Se recolectaron las notas publicadas por CRHoy en Facebook y sus respectivos comentarios gracias a un programa informático para esos fines (Netvizz); después se realizó un análisis cuantitativo y cualitativo a partir de una muestra de comentarios. Se observa que, de manera contra-intuitiva, la deliberación en las secciones de comentarios de CRHoy cumple con varios criterios formales de la teoría normativa del espacio público.

PALABRAS CLAVE: ELECCIÓN * POLÍTICA* DEBATE * OPINIÓN PÚBLICA* RED SOCIAL

\section{ABSTRACT}

This paper brings an analysis of public comments left by followers of costarrican news site CRHoy, in the aftermath of Gonzalo Ramirez's election as President of Congress. It looks to identify implicit norms and codes of online expression regarding public affairs, as well as to describe the main tendencies within the spontaneously formed opinions of users. We gathered all publications of CRHoy on Facebook thanks to a free software app, Netvizz; we then performed quantitative and qualitative analyses based on a sample of comments. One rather counter-intuitive finding we make is that these comments comply with several requirements of normative theories of public space.

KEYWORDS: ELECTION * POLITICS * DEBATE* PUBLIC OPINION * SOCIAL NETWORKS 


\section{INTRODUCCIÓN}

El $1^{\circ}$ de mayo de 2017, después de tres rondas de votación y muchas peripecias en el congreso, fue electo Gonzalo Ramírez, pastor evangélico y diputado del Partido Renovación Costarricense, para presidir la Asamblea Legislativa. Apoyado por el Partido Liberación Nacional (pLN), fracción principal en el congreso que no presentaba candidato propio, Ramírez triunfó sobre su rival Ottón Solís, diputado del Partido Acción Ciudadana ( $\mathrm{Pac}$ ), el cual contaba con el apoyo de una heterogénea coalición entre diputados del PAc, del Frente Amplio (FA) $y$ del Partido Unidad Social Cristiana (Pusc). Al final, Ramírez obtuvo 26 votos (contra 24 para Solís), a los cuales se sumaron siete votos inválidos de diputados del fa $y$ del pusc que, al votar por sí mismos, se negaron a respetar el acuerdo entre sus fracciones y Ottón Solís. Así quedó el pastor evangélico para presidir la Asamblea, después de un proceso relativamente caótico e inesperado, en un contexto marcado por la perspectiva de la elección presidencial del 2018.

Además de contar con una importante cobertura mediática, causada por la relevancia del cargo y la incertidumbre del proceso de votación, esta elección generó una gran cantidad de comentarios en las redes sociales, durante y después de la votación ¿Cómo reaccionaron las personas comentaristas en las redes sociales a esta elección? Así como lo plantean Gillespie (2014) o Boullier (2015), la expresión pública de los usuarios de las redes sociales constituye un material empírico sumamente interesante para las ciencias sociales, pues permite investigar la manera en que se estructura concretamente el espacio público en línea, en la medida en que recelan una inmensa cantidad de opiniones, pronósticos, críticas y de intercambios políticos. En este artículo, se propone entonces describir las discusiones públicas en las redes sociales en torno a la elección del nuevo presidente de la Asamblea, con el fin de contribuir al debate sobre el potencial democrático de Internet como medio de comunicación y de expresión.

Es cierto que este debate ha sido ampliamente documentado en la literatura de las ciencias sociales desde 19901; no obstante, estudios sobre el tema recaen en una oposición caricatural entre, por un lado, ensayos entusiastas sobre el rol de la tecnología, los cuales defienden el carácter democrático del Internet per se (Rheingold, 2000; Jones, 1995) —considerado como espacio igualitario de libre comunicación entre todos-, y por otro lado, estudios críticos que denuncian entre otros problemas, la violencia o la trivialidad de las discusiones entre anónimos y su fragmentación temática ${ }^{2}$ (Sunstein, 2001). Además, muchas veces la falta de investigaciones empíricas $y$ rigurosas tiende a limitar el análisis a una posición meramente normativa, a favor o en contra del efecto del Internet sobre nuestras sociedades democráticas (Beuscart, Dagiral y Parasie, 2016).

El presente artículo busca superar esta oposición estudiando el espacio público en línea tal como es, en sus formas técnicas y discursivas, indagando empíricamente la manera en que los usuarios de las redes sociales comentan, opinan y debaten sobre temas políticos. Al igual que Desrosières (2008), se defiende la idea de que el "espacio público" no es solamente un concepto filosófico abstracto, sino una realidad material, técnicamente producida $y$ mediada, en la cual las tecnologías tienen un papel decisivo: no es el mismo "espacio público" según está principalmente instanciado por los periódicos impresos, la televisión o las redes sociales; los protagonistas, los formatos, las reglas y la velocidad de producción de la información cambian radicalmente $y$ ejercen efectos muy concretos sobre el marco general del debate público. Estos últimos años, el surgimiento de una infinidad de microespacios de expresión en línea, le dio visibilidad a opiniones profanas, que de hecho desafían el monopolio de los medios tradicionales sobre el acceso a la publicidad (Cardon, 2010).

\footnotetext{
$1 \quad$ Ver por ejemplo Cardon (2010) o Flichy (2008) para una revisión de la literatura anglófona y francófona sobre el tema.

2 Hoy criticada como el efecto de "burbujas informativas", en las cuales cada individuo tiende a leer únicamente noticias y contenidos políticamente homogéneos.
} 
Este artículo se enfoca en los comentarios publicados por los usuarios de Facebook en las numerosas notas posteadas en esta plataforma por el sitio de información $\mathrm{CRHoy}^{3}$, dedicadas a la elección del nuevo presidente de la Asamblea Legislativa. En efecto, en estos comentarios participan muchos individuos, en un mismo espacio de libre expresión, alimentado por los contenidos noticiosos producidos por periodistas de CRHoy que proporcionan una base para la discusión. Más específicamente, corresponden a lo que Scott Wright (2012) define como "espacios terceros" de discusión y de socialización, los cuales se caracterizan por su poca formalidad y su facilidad de acceso. Así como lo plantea Wright, estos espacios fluidos e informales, que no son expresamente dedicados a la discusión política, se prestan muy bien para la expresión de opiniones y el diálogo: de manera análoga a lo que pasa en los bares o cafés populares, surgen discusiones políticas entre relativos desconocidos, sobre los temas agendados por los medios de comunicación (el periódico o la televisión en los bares, las notas de CRHoy en este caso). Los comentarios de la actualidad en Facebook son relevantes también por su carácter ordinario: a diferencia de muchos estudios dedicados a diferentes formas de activismo en línea ${ }^{4}$, interesa estudiar las conversaciones de un público más "ordinario" y no necesariamente muy politizado, en la continuidad de trabajos sociológicos (Monnoyer-Smith, 2006) e históricos (Farge, 1992).

Por supuesto, por sus características, las opiniones expresadas en las redes sociales no cumplen con los criterios estadísticos de la representatividad, ya que el acceso a Internet, el interés por la política y la propensión a opinar públicamente, son rasgos desigualmente distribuidos en la sociedad (Bourdieu, 1973). No se pretende entonces realizar un estudio representativo de las reacciones de las personas costarricenses a la elección de Gonzalo Ramírez, como lo haría una encuesta clásica. Sin embargo, las

$3 \quad$ Fuente de información en línea con más dinamismo en Costa Rica (ver el artículo de Siles et ál. en este volumen).

Cf. Wojcieszak (2008), Greffet y Wojcik (2008). tendencias que se expresan en estos comentarios interesan por dos motivos esenciales.

Primero, si bien la población de los comentaristas de CRHoy en Facebook podría no ser representativa de la sociedad en general, es suficientemente masiva para que su opinión no sea anecdótica o demasiada típica de una capa específica de la población. Luego y sobre todo, las opiniones que se expresan aquí son opiniones movilizadas, activamente elaboradas por usuarios suficientemente interesados o preocupados para expresarse públicamente y empezar a dialogar sobre el tema. Por lo tanto, ese material empírico permite acceder a los temas que más generan interés público.

En particular, meses antes del "Cementazo" y de las controversias sobre el matrimonio gay, que luego favorecieron el auge del candidato Fabricio Alvarado en la elección presidencial del 2018, se percibe muy claramente la importancia de los temas de corrupción, de sexualidad y de religión en las discusiones de las personas costarricenses en Facebook; cabe insistir sobre el hecho de que este análisis fue realizado a mediados del año 2017, sin saber la importancia que iban a tomar estos temas en el debate nacional. Parece entonces que, a pesar de que no se pueda asesar su representatividad con certeza, las opiniones que se publican en línea, por su espontaneidad y su alto nivel de movilización, proporcionan un acceso empírico inédito a las tendencias de fondo que trabajan una sociedad.

\section{ASPECTOS METODOLÓGICOS}

Gracias al programa Netvizz, concebido por el investigador holandés Bernhard Rieder (2013), se pudo captar la totalidad de las publicaciones de CRHoy en Facebook, durante una secuencia de cuatro días, del 30/04/2017 al 03/05/2017 (un día antes, el día de la elección de Ramírez y dos días después). El software también permitió capturar todos los comentarios posteados por los usuarios de Facebook en cada nota publicada por el sitio de información, así como ciertas métricas, como por ejemplo, la cantidad de likes y demás reacciones recibidas por cada nota informativa $y$ por cada comentario. Los comentarios, 
anonimizados $y$ organizados por nota, fueron indexados en una base de datos que después se ordenaron eliminando los que no tenían que ver con la elección del nuevo presidente de la Asamblea. Así se constituyó un corpus de 51 notas posteadas por CRHoy en Facebook, las cuales generaron 10299 comentarios de parte de los usuarios. Finalmente, se seleccionaron cuatro publicaciones entre las más comentadas, con enfoques variados para cubrir los diferentes aspectos de esta elección, las cuales se presentan en la siguiente tabla ${ }^{5}$ :

FIGURA 1

NOTAS ANALIZADAS DE CRHOY.COM

$1^{\circ}-3$ DE MAYO, 2016

\begin{tabular}{|c|c|c|c|c|}
\hline $\mathrm{N}^{0}$ & TÍTULO & CONTENIDO & $\begin{array}{l}\text { CANTIDAD DE } \\
\text { COMENTARIOS }\end{array}$ & $\begin{array}{l}\text { POSICIÓN } \\
\text { EN LAS } \\
\text { NOTAS MÁS } \\
\text { COMENTADAS }\end{array}$ \\
\hline 1 & $\begin{array}{l}\text { "Frente Amplio pide } \\
\text { renuncia de L. Fallas } \\
\text { y J. Arguedas" }\end{array}$ & $\begin{array}{l}\text { Artículo dedicado a las sanciones tomadas por el FA } \\
\text { en contra de estos dos diputados, que se negaron a } \\
\text { votar a favor de Ottón Solís a pesar del acuerdo entre } \\
\text { él y la fracción frenteamplista, y que así permitieron la } \\
\text { elección de Ramírez. }\end{array}$ & 379 & $5^{\mathrm{a}}$ \\
\hline 2 & $\begin{array}{l}\text { "Diputado: 'le } \\
\text { pusimos una lápida } \\
\text { a los derechos } \\
\text { humanos"” }\end{array}$ & $\begin{array}{l}\text { Artículo en el cual el diputado Edgardo Araya (FA) } \\
\text { deplora la agenda conservadora del nuevo presidente de } \\
\text { la Asamblea; también describe la estrategia equívoca del } \\
\text { PLN, que afirma "no apoyar" esa agenda mientras le dio } \\
\text { sus votos al pastor evangélico. }\end{array}$ & 265 & $8^{\mathrm{a}}$ \\
\hline 3 & $\begin{array}{l}\text { "Conozca al nuevo } \\
\text { presidente de la } \\
\text { Asamblea en nueve } \\
\text { frases" }\end{array}$ & $\begin{array}{l}\text { Nota que hace un listado de frases pronunciadas por G. } \\
\text { Ramírez que representan su ideología política. Estas } \\
\text { frases se refieren principalmente a los temas de familia } \\
\text { y derechos sexuales. }\end{array}$ & 236 & $9^{\mathrm{a}}$ \\
\hline 4 & $\begin{array}{l}\text { “Cómo sería el } \\
\text { Congreso si Ottón } \\
\text { Solís lo liderara?" }\end{array}$ & $\begin{array}{l}\text { Artículo previo al voto, dedicado a las propuestas del } \\
\text { candidato Ottón Solís, principalmente en relación } \\
\text { a la reducción del gasto público, inclusive en el } \\
\text { funcionamiento de la misma Asamblea. }\end{array}$ & 110 & $24^{\mathrm{a}}$ \\
\hline
\end{tabular}

Fuente: Elaboración propia.

5 Las notas se pueden encontrar en las siguientes URL: https://www.CRHoy.com/nacionales/frente-ampliopide-renuncia-de-jorge-arguedas-y-a-ligia-fallas/ https://www.CRHoy.com/nacionales/diputado-le-pusimos-una-lapida-a-los-derechos-humanos/ https://www.CRHoy.com/nacionales/conozca-alnuevo-presidente-de-la-asamblea-en-9-frases/ https://www.CRHoy.com/nacionales/como-seriael-congreso-si-otton-solis-lo-liderara/ 
Se leyeron y codificaron individualmente cada comentario dejado por los usuarios de Facebook en estos cuatro artículos, o sea 1135 comentarios; se eliminaron los comentarios vacíos o incomprensibles fuera de contexto y se llegó a un corpus final de 990 comentarios codificados. Los criterios de la codificación, inspirados por el trabajo de Parasie y Cointet (2012), fueron definidos primero con el fin de describir sintéticamente el contenido de cada comentario, caracterizando los principales temas del comentario, la referencia (o no) a otro tema no mencionado en la nota de CRHoy, $y$ la "tonalidad" del comentario en relación a la elección de Ramírez y sus protagonistas (positiva, neutra 0 negativa). Una segunda serie de criterios buscaba caracterizar el espacio público materializado por estos comentarios, analizando los modos de expresión de los comentaristas. Se codificó la presencia (o ausencia) de los siguientes elementos en cada comentario:

$\diamond \quad$ Referencia a experiencias personales

$\diamond \quad$ Argumentación a favor de una solución o acción colectiva en relación al asunto debatido

$\diamond \quad$ Voluntad de revelar elementos o causalidades nuevas para explicar la situación debatida

$\diamond \quad$ Diálogo con otros comentaristas

A partir de este análisis cuantitativo $y$ cualitativo, se propone primero un estudio de la estructura del espacio de discusión, tal como se materializa en los comentarios analizados, confrontándolo con los criterios más clásicos y socialmente legítimos del espacio público, tal como son definidos por la filosofía política de Jurgen Habermas (1999) ${ }^{6}$. A pesar de los prejuicios que uno puede tener en relación a las discusiones online, los códigos y normas tradicionales de la expresión pública permanecen significativamente presentes. Luego se estudia

$6 \quad$ A diferencia de muchos trabajos sobre el espacio público, el de Habermas tiene el interés de no enfocarse solamente en los medios de comunicación, sino que plantea varios criterios normativos para la deliberación democrática, o sea, la participación de todos en la esfera pública. en un modo más cualitativo cómo se desarrollan opiniones, y sobre todo críticas, en relación a la elección de Ramírez; se enfoca en los temas $y$ las tendencias principales de las publicaciones generadas durante esta secuencia política, demostrando una relativa autonomía de la esfera pública de los comentaristas, la cual promueve algunos temas independientemente de la agenda mediática.

\section{FORMATOS DEL ESPACIO PÚBLICO EN LÍNEA: INDIVIDUALIDAD, DIÁLOGO Y CIVISMO}

Antes que todo cabe destacar el dinamismo $y$ la productividad de este espacio de discusión política. Tal y como se dijo anteriormente, en las 51 notas publicadas por CRHoy en $\mathrm{Fa}$ cebook sobre la elección de Gonzalo Ramírez, los lectores dejaron un total de 10299 comentarios, ya sea un promedio de 201 comentarios por nota y una mediana de 128. Como lo indica el siguiente gráfico, la nota con más comentarios obtuvo 2151 y la menos comentada obtuvo solamente cuatro ${ }^{7}$.

\footnotetext{
$7 \quad$ Respectivamente: "Ligia Fallas y sus eternas "chanclas' son ajenas a las críticas" (01/05/2017) y "Ottón Solís y Gonzalo Ramírez disputarán elección en tercera ronda” (01/05/2017).
} 
FIGURA 2

CANTIDAD DE COMENTARIOS POR NOTA

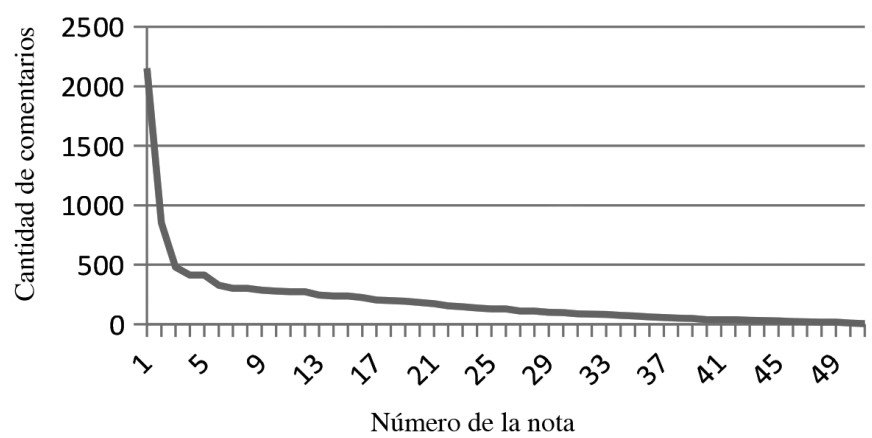

Fuente: Elaboración propia.

Esta primera constatación denota el interés de los seguidores de CRHoy en $\mathrm{Fa}$ cebook por esta elección, lo cual puede sorprender en una ocasión tan institucional como la renovación anual del directorio de la Asamblea Legislativa. Eso sí, el carácter indeciso de la votación, el contexto preelectoral y las alianzas poco esperadas como la de Liberación con los cristianos o del FA con el pusc y el pac hicieron especial esta elección; también se verá en la segunda parte de este artículo que esta profusión de comentarios corresponde a varios movimientos propios de los comentaristas. Pero antes de esto, se propone analizar la manera en que se estructura el espacio de la discusión, sus reglas y sus formas de expresión, a partir de la siguiente tabla de resultados:

FIGURA 3

PROPIEDADES DEL ESPACIO PÚBLICO EN LÍNEA

\begin{tabular}{ccccc}
\hline & EXPERIENCIA & \multirow{2}{*}{ CONVERSACIÓN } & \multicolumn{2}{c}{ POSTURA CÍVICA* } \\
\cline { 4 - 5 } & PROPIA & & REVELACIÓN & COLECTIVO \\
\hline Valor absoluto & 24 & 225 & 125 & 170 \\
Porcentaje & $2,40 \%$ & $22,70 \%$ & $12,60 \%$ & $17,20 \%$ \\
\hline
\end{tabular}

Fuente: Elaboración propia.

Nota *: Se analiza detalladamente cada categoría de esta tabla en las páginas siguientes.

\section{LA EXTREMA ESCASEZ DE LAS EXPERIENCIAS INDIVIDUALES}

En los comentarios se buscaron primero las referencias a experiencias personales: ¿usa o no el autor elementos de su vida cotidiana, de su experiencia propia o de su historia individual (o de sus familiares)? De esta manera, se quiso averiguar la importancia relativa de los elementos biográficos, tal como la situación profesional, familiar, etc., en los argumentos elaborados por los comentaristas. En efecto, un criterio esencial de la discusión pública definido por autores como Jurgen Habermas recae en el hecho de que los participantes se deshagan lo más que puedan de sus particularismos, de sus puntos de vista personales, para alcanzar cierto grado de generalidad en sus argumentos.

En este aspecto, cabe denotar la manera en que los comentaristas de CRHoy se conforman con esta condición de la discusión racional: de los 990 comentarios codificados, 
solamente 24 se refieren a la experiencia del autor como persona, o sea, un $2,4 \%$ del total; en ninguna de las cuatro notas analizadas esta proporción supera el 4\%. Los participantes de la discusión pública en las notas posteadas por CRHoy en Facebook comentan entonces a partir de sus opiniones $y$ análisis propios, pero no utilizan su trayectoria individual como base argumentativa.

De esta manera, se alinean con la exigencia del universalismo que caracteriza el espacio público en su definición clásica; de igual forma, este resultado contradice algunos clichés y prejuicios sobre la discusión en línea, la cual consistiría en una acumulación de puntos de vista individuales - centrados en la persona que se expresa $-y$ hasta triviales. Al contrario, si bien la subjetividad está constantemente presente, la casi ausencia de este tipo de elementos biográficos demuestra que los autores de los comentarios recurren muy poco a su situación particular para opinar sobre asuntos públicos, por lo menos en este tema de política institucional.

\section{UN ESPACIO RELATIVAMENTE CONVERSACIONAL Y CONFLICTIVO}

Posteriormente, se procuró investigar la proporción de comentarios que se inscribían en una dinámica de diálogo, buscando marcas de interpelación (i.e. mención del nombre de otra persona) o respuestas directas a argumentos previamente expresados. Se buscó identificar las formas de expresión que prevalen en los comentarios analizados, viendo en qué medida los comentarios hablan por ellos mismos, en una forma de monólogo, o más bien en una perspectiva de intercambio o de argumentación con otra persona, lo cual también constituye un criterio de la discusión pública según sus definiciones clásicas.

Se observa en la figura 3 que la conversación en sí no es tan frecuente en los datos analizados, aunque aquí el resultado sea probablemente subestimado: la base de datos en la cual se trabajó no permitía distinguir la arborescencia de los comentarios con sus respectivas respuestas, tal como aparece en Facebook. Alrededor de un $23 \%$ de los comentarios analizados corresponden a algún tipo de discusión; estos intercambios pueden ser de un alto grado de desarrollo y hasta de especialización temática según los asuntos debatidos.

Dos tipos de diálogos se observan en torno a las notas de CRHoy. El primero sería una forma sociable o amistosa de conversación. Son interpelaciones de otra persona, que corresponden a los mecanismos propios de la plataforma Facebook: el hecho de "etiquetar" a un amigo (escribiendo su nombre en el comentario) hace que él reciba una notificación en su página $y$ se entere del comentario $y$ de la publicación a la cual está vinculado. Por ejemplo, un tipo recurrente de comentarios que sirven para compartir un contenido, o aún más una opinión al respecto, como este formulado a propósito del Frente Amplio: "Andrés Zárate. que asco de partido" 8 . Este tipo de comentarios crean generalmente conversaciones bastante cortas, inscritas en una sociabilidad preexistente entre sus participantes.

El segundo tipo de diálogo, mucho más frecuente, corresponde a las conversaciones conflictivas o por lo menos antagonistas, en las cuales usuarios que no se conocen intercambian y se responden a partir de comentarios ya posteados. Este modo de conversación es mucho más conflictivo, ya que el anonimato relativo de sus protagonistas limita el poder de las convenciones tradicionales de interacción entre desconocidos (Goffman, 1982). Ese tipo de conversación surge con mayor fuerza en los temas de diversidad sexual, religión, derechos humanos y familia, los cuales pueden suscitar comentarios de descrédito, ambos bandos calificándose con términos abiertamente peyorativos. No obstante, estas conversaciones no se pueden reducir a un intercambio de palabras ofensivas: muchas veces los comentaristas se corrigen mutuamente, aportan elementos nuevos o marcan su aprobación, como en el tercer comentario de la siguiente lista de ejemplos:

\footnotetext{
$8 \quad$ Nota $n^{\circ} 1-03 / 05 / 2017$. El texto en negrita es originalmente cliqueable y corresponde al mecanismo de "etiquetar" al amigo. Todos los apellidos que aparecen en el artículo fueron cambiados; se deja la ortografía tal como aparecía en los comentarios.
} 
$\diamond \quad$ "Usted no sabe un pepino sobre cómo funciona la economía; gracias al pobre intelecto del pueblo ( $y$ diputados en especial), es que este país no avanza"9.

$\diamond \quad$ "Carlos Bueno vuelva a su cueva cavernícola"10.

$\diamond \quad$ "Danilo Araya. Tiene razón. La ignorancia se desborda. La fecundación "IN VITRo" ya se está realizando. La ley de maltrato animal, fue analizada y rechazada por la sala iv. Para qué quiere una ley que puede ser declarada inconstitucional?"11.

Aquí se observa el potencial de controversia asociado con estos temas de diversidad sexual y de derechos humanos, constatando que la nota $n^{\circ} 2$ de este corpus, que relata las críticas de Edgardo Araya hacia la agenda conservadora del nuevo presidente, tiene una proporción mucho mayor de comentarios "conversacionales" que las demás: 34\% de los comentarios asociados a esta nota se inscriben en algún tipo de intercambio, contra solamente $15 \%$ en el caso de las notas $n^{\circ} 1$ y 4 , y $24 \%$ en el caso de la nota $n^{\circ} 3$. Como se verá posteriormente, estos temas son muy importantes para los comentaristas de CRHoy; son los que más generan controversia.

La conversación en sí, que según las notas constituyen un cuarto o un tercio de los comentarios, se define entonces por su relativo antagonismo, lo cual concuerda con el carácter generalista de CRHoy, que se dirige a un público políticamente heterogéneo. Sin embargo, aunque haya conflictividad en estos intercambios, los insultos son muy pocos y siempre corresponden a los mensajes con menos likes y por lo tanto, los menos visibles según las reglas propias de Facebook. El espacio constituido por las notas de CRHoy, relativamente neutro desde el punto de vista político, permite entonces la emergencia de un espacio de intercambio antagónico que corresponde a otra exigencia del debate público en democracia.

\footnotetext{
$9 \quad$ Nota ${ }^{\circ} 4-01 / 05 / 2017$

$10 \quad$ Nota $^{\circ} 2-02 / 05 / 2017$

$11 \quad$ Nota $^{\circ} 2-02 / 05 / 2017$
}

LA PERMANENCIA DE LOS CÓDIGOS DEL ESPACIO PÚBLICO: UNA POSTURA CÍVICA

Como último modo de caracterización de este espacio público, se investigó el peso de lo que se llama una postura "cívica", definida como voluntad manifestada por los comentaristas de debatir o de actuar de manera colectiva, como ciudadanos y no simplemente como individuo opinante. Dos variables definen esta postura que corresponde a la actitud más esperada y legítima de comportarse en el espacio público: la búsqueda racional $y$ argumentada de la verdad a propósito de los asuntos públicos, y la elaboración de respuestas colectivas aplicables para un problema definido.

Esta postura se define como el conjunto de dos variables: primero, se codifican los comentarios caracterizados por una postura investigativa, denotando una voluntad de parte del comentarista de revelar a los demás elementos ignorados o causalidades nuevas para contextualizar, interpretar o explicar el asunto debatido; los comentarios que corresponden a la columna "Revelación" (ver figura 3) son entonces los que quieren aportar hechos no mencionados en la nota de CRHoy, que pretenden develar elementos considerados como relevantes pero ignorados por el público, o que buscan factores explicativos más allá del contenido de la nota periodística. Inspirada por la sociología pragmática de Lemieux (2007) o de Boltanski (2009), esta variable permite visibilizar las competencias críticas de los actores y estudiar la manera en que confrontan interpretaciones $y$ justificaciones para producir contradictoriamente la realidad social (en este caso, los hechos que componen la "actualidad política", o sea el marco de la deliberación).

Y no olviden que [G. Ramírez] es un exconvicto condenado por falsificar la firma de un juez para el traspaso de unas propiedades en el 2001 y el colegio de abogados le quitó las credenciales de notario por eso en el año 2008, pero bueno que se puede esperar del PLN? Entre riñas y mafias se entienden ${ }^{12}$.

$\overline{12 \quad N_{0}}{ }^{\circ} 3-02 / 05 / 2017$ 
No fue exconvicto, ni hubo sentencia condenatoria sino una conciliación como dice una noticia de CRHoy. No lo defiendo pero no se puede basar en mentiras ${ }^{13}$.

La segunda variable, identificada en la figura 3 como "Colectivo", corresponde a los comentarios que llaman a alguna forma de acción colectiva, o sea, en los que sus autores hacen propuestas de políticas públicas, requieren algún tipo de cambio en las instituciones o llaman a sus pares a actuar de determinada forma en el futuro. Este segundo criterio permite identificar los discursos normativos de largo alcance, dirigidos a la ciudadanía en general, que no solamente critican un estado de las cosas, sino que también proponen soluciones, ubicándose deliberadamente dentro del registro de la esfera pública:

Gente! Por favor tenemos que salir a votar, pero también hay que cuidar los votos trabajando en las mesas y convenciendo a todos esos que piensan que no es importante votar!!!! No MAS PLN ${ }^{14}$.

Por eso hay que variar la forma de escoger los diputados. La propuesta de poder ciudadano ya, es que tiene que darse a conocer ${ }^{15}$.

Agregando estas dos variables ("Revelación" y "Colectivo"), es posible entonces definir una postura "cívica" que corresponde a la forma de contribución más elevada en el espacio público en sus criterios tradicionales, como búsqueda del bien común. Es muy interesante constatar que esta postura se encuentra presente en los comentarios de CRHoy en Facebook: un $12,6 \%$ de los comentarios se caracteriza por su voluntad de aportar o revelar hechos nuevos no mencionados en las notas comentadas, mientras que un $17,2 \%$ defiende explícitamente

$13 \quad$ Nota $n^{\circ} 3-02 / 05 / 2017$

$14 \quad \operatorname{Nota}^{\circ} 3-02 / 05 / 2017$

$15 \quad$ Nota ${ }^{\circ} 3-02 / 05 / 2017$ algún tipo de solución política en cuanto al asunto debatido.

Si se incluyen estas dos posturas, se observa que 267 comentarios contienen una postura de revelación o una propuesta colectiva (27\% del total de los comentarios analizados). De igual forma, un 2,8\% de los comentarios consiste en una postura cívica "dura" que simultáneamente se ubica en ambos registros, es decir, un poco más del total de los comentarios que mencionan experiencias propias. Si bien, la postura cívica no es mayoritaria en lo que publican los seguidores de CRHoy en Facebook, existe en proporciones significativas.

Como lo demuestra este breve análisis cuantitativo, en el espacio de debate delimitado por los comentarios de CRHoy en Facebook, permanecen los códigos más tradicionales de la esfera pública: primero se observa que casi todos los comentaristas renuncian a argumentar en base a sus experiencias personales $y$ por otro lado, se constata la existencia de fracciones significativas de comentaristas caracterizadas por su inscripción en la gramática clásica de la ciudadanía, confrontando opiniones, proponiendo interpretaciones de la situación política y buscando remedios y soluciones para mejorarla.

La discusión en Facebook a propósito de asuntos políticos, tales como la elección del nuevo presidente de la Asamblea Legislativa, implica en realidad un grado bastante elevado de "seriedad" cívica que no se deja resumir al cliché del debate político en línea como conjunto de trivialidades, reino de los "trolls" y de los argumentos ad hominem. Por supuesto, la discusión en línea está muy lejos de ser puramente racional y cívica; no obstante, este análisis preliminar tiende a demostrar que sería un error considerar este material como trivialidades. Al contrario, parece que hay que interesarse más específicamente a las tendencias de opinión que circulan en estas discusiones.

\section{TENDENCIAS Y MOTIVOS DE LA OPINIÓN EN LÍNEA: UNA AGENDA RELATIVAMENTE AUTÓNOMA}

Después de haber analizado las formas y los códigos de la expresión pública en los comentarios de CRHoy, se debe estudiar específicamente su 
contenido, los temas más debatidos y la medida en que se relacionan con la agenda mediática. Lo que arroja el primer análisis de contenido de las opiniones expresadas en Facebook es su negatividad: un $69 \%$ de los comentarios codificados aparecen como desfavorables a la elección de Gonzalo Ramírez, por una serie de razones distintas o por sus protagonistas (PLN, diputados del FA que votaron nulo, etc.); un 15,4\% de los comentarios aparecen como positivos, mientras que un $15,7 \%$ no denota específicamente un sentimiento al respeto $y$ fue categorizado como "neutro"16.

Pero, más allá de esta polarización mayoritariamente negativa, ¿de qué hablan los comentaristas? En esta segunda parte, de manera más cualitativa, se demuestra la relativa autonomía de la esfera de los comentaristas frente a la agenda de CRHoy. En efecto, los temas que más apasionan al público y que generan debate, no son exactamente los mismos que los desarrollados en las noticias: un 54\% de los comentarios analizados mencionan por lo menos un tema ausente de la nota que comen$\tan$ (nuevos protagonistas, enfoques distintos, etc.). A continuación, se presentan tres grandes categorías de críticas que hacen los comentaristas a los protagonistas de la elección de Gonzalo Ramírez, cada cual conduciendo a un discurso de alcance más general sobre la sociedad costarricense.

\section{ANTECEDENTES JUDICIALES DE GONZALO RAMÍREZ Y DENUNCIA DE LA CORRUPCIÓN POLÍTICA}

Una primera crítica en contra del nuevo presidente de la Asamblea Legislativa consiste en reprocharle su supuesta corrupción. Así,

$\overline{16}$ Por cierto, no todos los comentarios pueden fácilmente ser categorizados como positivos, neutros o negativos: algunos usan ironía, lo cual es a veces muy difícil de detectar por escrito; otros son complejos y no se dejan reducir en términos de positividad o negatividad. Como consecuencia, los números producidos aquí deben ser considerados con prudencia y no pretenden tener un estatus de resultado definitivo. Su propósito es brindar una idea general de la apreciación de los comentaristas en torno a los eventos estudiados. muchos comentarios, juntos con las notas de CRHoy y de varios medios de comunicación, mencionan los antecedentes del diputado evangélico con la justicia. En el 2001, Gonzalo Ramírez fue detenido dos meses por prisión preventiva, al ser sospecho de falsificación de la firma de juez de Heredia en un caso de adquisición de terrenos; aunque este caso haya terminado en conciliación y nunca llegó a juicio, son muchísimos los comentaristas que recuerdan estos hechos $y$ acusan al nuevo presidente de corrupción, inclusive cuando la nota que están comentando no mencione estos hechos.

Ramírez estuvo en prisión preventiva por dos meses, en el año 2001, cuando se le acusaba por la aparente falsificación de la firma de un juez, pero también, por supuesta asociación ilícita, fraude de simulación y falsificación de documentos ${ }^{17}$.

En 2009, Telenoticias publica serie de reportajes sobre iglesia "Pasión por las Almas", incluyendo investigación con cámara escondida. Los titulan "Pasión por el dinero" y explican cómo pastores que no tienen estudios formales en teología, pasan a tener a su nombre propiedades valoradas en millones de dólares, carros Audi y hasta jet privados. En el primer reportaje aparece el video del pastor Gonzalo Ramírez predicando en esa iglesia ${ }^{18}$.

Como conozca? Es que creen que no es conocido, pues si se le conoce y no precisamente por cosas buenas, sino escándalos, corrupción y porquerías como las de todos en el pln. Y se dice pastor lo que da es vergüenza. Por favor no le hagamos esto al país. NO MAS LIBERACIÓN NACIONAL ${ }^{19}$.

\begin{tabular}{ll}
\hline 17 & Nota $n^{\circ} 3-02 / 05 / 2017$ \\
18 & Nota $n^{\circ} 4-01 / 05 / 2017$ \\
19 & Nota n ${ }^{\circ} 3-02 / 05 / 2017$
\end{tabular}


Como se puede observar, la opinión en torno a la persona de Gonzalo Ramírez es bastante negativa y una fracción extensa del público de CRHoy no le perdona su involucramiento en asuntos de falsificación, a pesar de que nunca fuera condenado por ello. Además, una parte de estas críticas por corrupción se combinan con las responsabilidades religiosas del nuevo presidente de la Asamblea: el contraste entre los hechos que se le imputan a G.Ramírez y su papel moral de pastor evangélico produce críticas particularmente severas de su persona, que giran alrededor de los temas de la hipocresía y de la duplicidad, utilizando palabras como "chusma" o "fichita", $y$ que denuncian la falsa moralidad de un personaje que predica una cosa y hace otra. $\mathrm{Mu}$ chos de sus autores, cristianos reivindicados, ubican explícitamente su crítica en el campo religioso: los cargos contra G. Ramírez son según ellos, incompatibles con el hecho de ser un "buen cristiano".

Sin embargo, lo más interesante es la manera en que, partiendo de los hechos imputados al pastor evangélico, se agregan elementos nuevos que conducen a una crítica más general de la corrupción política en Costa Rica, la cual meses antes de que estallara el "Cementazo", preocupaba a los participantes en los comentarios de las noticias. Una gran cantidad de ellos usan términos virulentos contra la clase política del país, denunciando a los políticos "choriceros" o la supuesta avidez de diputados más preocupados por su curul que por el bien común. Así, comentando las propuestas de Ottón Solís para la reducción de gasto en la Asamblea Legislativa, un comentarista escribe: "Imposible [que se adopten estas reformas], no les conviene a los vividores que les recorten tantos beneficios. En este país un hombre tan honrado jamás quedará de Presidente"20.

En estas narrativas producidas por los comentaristas, la honestidad aparece como una desventaja en una competición política marcada por las trampas y las maniobras dudosas. Un elemento interesante de este desplazamiento de la crítica más allá de la persona de Gonzalo
Ramírez es que el partido más mencionado por los comentaristas anticorrupción es el pLN, considerado como principal responsable de su elección. Comentarios recurrentes llaman explícitamente a la desaparición de un partido que describen como emblemático de los asuntos de corrupción en el país; al respecto, la memoria de los escándalos de los años 1990 parece seguir viva.

\section{RELIGIÓN, DIVERSIDAD SEXUAL Y CONSERVADURISMO}

Meses antes de los debates en torno a la decisión de la Corte Interamericana de Derechos Humanos sobre el matrimonio gay $y$ de la accesión de Fabricio Alvarado a la segunda vuelta de la elección presidencial, se percibe en los comentarios analizados, como los temas de sexualidad y de religión generan controversia e interés público. El hecho de que un pastor evangélico acceda a la presidencia del primer poder de la república genera primero muchas críticas de parte de los comentaristas, por razones diferentes. Una primera categoría de comentarios denuncia la implicación de la religión en los asuntos del gobierno; muchos consideran anormal que sea posible para un pastor ejercer este cargo. En los comentarios, este tipo de crítica muchas veces se asocia con reivindicación de un Estado laico, en el cual se prohibiría a cualquier responsable religioso ejercer un cargo público.

Asco da. La política y la religión son dispares. Necesitamos en Costa Rica estado laico. El fanatismo religioso es peligroso, parcializado y solo acepta su fe como verdad absoluta. Un mercader de la fe y abogado corrupto, no merece semejante distinción. Liberación ha orquestado con esta patraña, su entierro para las próximas elecciones ${ }^{21}$.

La agenda conservadora de Gonzalo Ramírez, explícitamente basada en su religión, es el elemento que más causa controversias, 
en los temas de familia $y$ de derechos sexuales (igualdad de género, derecho al aborto, matrimonio gay, fecundación in vitro, etc.). Estos temas, como se vio anteriormente, generan gran debate entre los comentaristas, muestran una oposición extremadamente marcada entre detractores $y$ defensores de la agenda explícitamente conservadora del nuevo presidente de la Asamblea. En línea con las declaraciones del diputado frenteamplista Edgardo Araya, reportadas en la nota $n^{\circ} 3$ del corpus, muchos comentarios denuncian el posicionamiento conservador de Gonzalo Ramírez en los temas de género, familia $y$ diversidad sexual; su elección a la presidencia del poder legislativo es considerada como mala señal para los derechos humanos en estos ámbitos.

[...] Estamos hablando de equidad de género que ese señor tanto ha humillado en la asamblea, estoy hablando de derechos animales, estoy hablando libertad religiosa no fanatismo, estoy hablando de derechos igualitarios en matrimonio $y$ patrimonio $y$ seguridad social, estoy hablando de derechos ambientales que ese partido y muchos más han detenido 22 [...].

Al contrario, otro bando de comentaristas, a pesar de ser minoritario en los datos analizados, reacciona ante este tipo de acusación. Presentándose como garantes de la moral y basados en sus convicciones religiosas, ellos se dedican a responder de manera detallada a los comentarios de los adversarios de Ramírez, hasta crear secuencias largas de discusión más o menos virulentas con recursos argumentativos variados, ya sean religiosos ${ }^{23}$, morales o culturales.

Como se puede ver en los siguientes ejemplos, un punto esencial de la controversia radica en el concepto de derechos humanos: en efecto, los partidarios del nuevo presidente desarrollan un esfuerzo argumentativo amplio y sistemático para desconectar los temas de

$22 \quad$ Nota $n^{\circ} 2-02 / 05 / 2017$

23 Muchas veces aparecen recomendaciones de leer algún pasaje de la Biblia y hasta citas integrales. diversidad sexual, aborto e igualdad de género de la carga simbólica positiva y poco contestable que conlleva este término. Dedican entonces mucha atención en afirmar y repetir que el matrimonio gay o el derecho al aborto "no son derechos humanos".

Lo que quieren es el derecho de estar en libertinaje $y$ hacer las perversidades que tienen en la mente ver a sus hijos hombres casados con otro hombre o mujer con una mujer, todos en Costa Rica tenemos los mismos derechos pero ellos quieren tener derechos que no van con los principios que Dios nos da ${ }^{24}$.

La palabra derechos humanos está secuestrada por la comunidad Gay y se ha vuelto un cliché que repiten como loros exigiendo las cosas con malacrianzas y sin un diálogo por lo menos un poquito racional. Entiendan que el universo no gira alrededor de ustedes, hay derechos humanos por encima de lo que piden y que involucra a más personas $y$ no a un grupo ${ }^{25}$.

Este tipo de comentarios genera a su vez réplicas de parte del bando opuesto, el cual afirma el carácter "retrógrado" e irrelevante de tales consideraciones. Las oposiciones reveladas por este conjunto de temas y los enfrentamientos que producen, parecen estar profundamente arraigados en la sociedad costarricense, ya que la conversación se propaga muy rápidamente al tocar estas temáticas. A través de estas discusiones se confrontan visiones opuestas de la religión cristiana y del peso que puede legítimamente ejercer sobre la sociedad costarricense.

\section{CRÍTICA DE LAS ESTRATEGIAS PARTIDARIAS DEL PLN Y DEL FA}

El último gran motivo de descontento entre los comentaristas de CRHoy en este

$\begin{array}{ll}24 & \text { Nota } n^{\circ} 2-02 / 05 / 2017 \\ 25 & \text { Nota } n^{\circ} 2-02 / 05 / 2017\end{array}$


tema es la actitud de dos partidos políticos en la elección de Gonzalo Ramírez: Liberación Nacional y el Frente Amplio. El primero es claramente el partido más criticado en todos los comentarios analizados, en las cuatro notas del corpus (que se refieran o no al pun de manera explícita). Una parte de las críticas que se dirigen a este partido adoptan el esquema explicativo mayoritario entre los diferentes medios de comunicación durante esta elección: la fracción liberacionista en la Asamblea, bajo la supervisión del candidato presidencial Antonio Álvarez Desanti, renunció en presentar un candidato propio y apoyó a G. Ramírez en un movimiento estratégico destinado a enviar una señal al electorado cristiano del país, y captar sus votos para la elección presidencial del 2018. Por esta concesión realizada a favor de la agenda conservadora del partido evangélico, en el marco de una estrategia de conquista del poder ejecutivo, se le reprocha a Liberación Nacional una forma de oportunismo político.

Como se vio anteriormente, muchos de los comentarios llaman a que desaparezca este partido del juego político costarricense; una proporción importante de ellos predicen la derrota severa del pLn en el 2018 causada por esas maniobras: "el pLn selló hoy su sentencia de derrota de las elecciones presidenciales" ${ }^{26}$; "el PLN prefirió apoyar a un fanático religioso exconvicto antes de apoyar al Pac. El PLN ha cavado su propia tumba para el 2018"27.

El Frente Amplio es el otro partido que se encuentra en el blanco de las críticas. Una primera categoría de comentarios se enfoca particularmente en el caso de Ligia Fallas y de Jorge Arguedas, los dos miembros de la bancada frenteamplista que se negaron a darle su voto a Ottón Solís, a pesar del acuerdo entre las fracciones de fa y del pac. Se les reprocha a los dos diputados su egoísmo y su falta de lealtad partidaria, que indirectamente permitió la elección de Gonzalo Ramírez ${ }^{28}$. Pero la crítica del fa va

\footnotetext{
$26 \quad$ Nota $n^{\circ} 2-01 / 05 / 2017$.

$27 \quad$ Nota $n^{\circ} 3-02 / 05 / 2017$.

28 Ambos votaron por sí mismos a pesar de no ser candidatos oficiales para la presidencia de la Asamblea, lo cual según el reglamento de la
}

mucho más allá de los acontecimientos en el congreso: un tema recurrente en los comentarios de las dos notas que mencionan el fa es la cuestión de la relación entre el Frente Amplio $y$ los gobiernos de izquierda en América Latina. En este tema, es impresionante la cantidad de comentaristas que se refieren a Venezuela, Cuba o Nicaragua, aunque estos países no hayan sido mencionados ni mínimamente en las notas periodísticas de CRHoy.

Esteban Herrera Claro que me preocupo por Costa Rica...por eso partidos como el FA que admiran ideologías fracasadas deben de ser desenmascarados...que opinaría ud de una candidata del fa para vicepresidente de la Republica diciendo que "prefiere la Venezuela de hoy"??? O sea... importar el fracaso de otros paises al nuestro? Por eso debemos ver los horrores de los fracasados en otros lados para nunca traerlos a Costa Rica $[\ldots]^{29}$.

Muchos diputados y diputadas hablan $y$ hablan del socialismo $y$ aplauden a Maduro. Pero no sueltan el vil veneno del salario (incentivo capitalista)... HIPócritas. Vayan a Cuba a vivir con menos de un dólar al día, no van...... HiPócritas... ${ }^{30}$

Más allá de la actitud de los diputados frenteamplistas durante el $1^{\circ}$ de mayo, el debate se concentra más en saber si se puede comparar este partido con los regímenes que se reivindican del socialismo en América Latina. Se discute sobre los méritos de dichos gobiernos y sobre la oportunidad de tomarlos como "modelo" para Costa Rica. Claramente, las dificultades económicas con las que se enfrentan estos países en los últimos años, particularmente en el caso actual de Venezuela, generan una gran desconfianza por parte de muchos comentaristas,

Asamblea Legislativa cuenta como voto nulo y se agrega a los votos del candidato con más sufragios, en este caso, G. Ramírez.

$30 \quad$ Nota $^{\circ} 2-01 / 05 / 2017$ 
los cuales comparan al Frente Amplio con la revolución bolivariana y por lo tanto, advierten al público sobre el "peligro" que representa este partido para el país. Respondiendo a estos argumentos, los partidarios del Frente Amplio se esfuerzan en circunscribir el debate, restringiéndolo al caso de Costa Rica ("Le repito hableme de Costa Rica no de venezuela ni otros pais, detras de un voto en contra hay mucha mas informacion y se analiza situaciones de las que usted no se entera" ${ }^{31}$ ).

Por fin, se observa en algunos casos que el debate se amplía aún más allá de las relaciones entre las izquierdas latinoamericanas, para llegar a la cuestión del comunismo. De hecho, muchos comentarios asocian también el Frente Amplio a la ideología comunista, la mayoría de ellos manifestando un anticomunismo radical ("Comunistas socialistas=miseria e ignorancia"32). Este tipo de comentario genera reacciones de otros comentaristas que buscan defender los avances sociales permitidos por los comunistas a lo largo de los años, en especial en el caso de Costa Rica, lo cual produce a su vez reacciones $y$ debates especializados sobre la historia de las garantías sociales y el papel del comunismo en estas.

Marvin Rodríguez Hay un libro de este señor historiador Vladimir de La Cruz en donde se clarifica conceptos erroneos como decir que la conquitas sociales son exitos del socialimos/comunismo/ similares...... la primera es que no fueron los comunistas que implantaron los beneficios sociales ( el partido comunista nunca ha tenido una presidencia en Costa Rica ), Manuel Mora propuso una serie de reformas inspiradas en organizaciones gremiales de usa y Europa Occidental con tal de ganar una base fiel o agradecida para poder perpetuar el Calderonismo al estilo Batista o Somoza (dijo: "Señor presidente, si les damos esto, nunca nos darán la espalda" ), una vez aprobado por el congreso (en donde
ToDos los partidos votaron), se establecieron las bases de nuestra seguridad social, pero con el fin primario de amenazar a los pobladores de esa época que si otro gobierno que no fuera Calderonista llegara al poder, todo eso se iba a perder. El Calderonismo se alimentó militarmente de los socialistas de America Latina ya que vieron una oportunidad de permear el comunismo en Costa Rica. Cuando Otilio Ulate quedó presidente - antes los atónitos sociales,--fiel a su estilo de imponer en vez de convencer,$- y$ dijeron "aca no le damos el poder a nadie" y nombraron a Calderon Guardia como ganador lo que desencadenó la famosa guerra del $48^{33}$.

Muy sierto Don Rodrigo Morales yo ya cumpli 60 años por esos mismo comunista se fue la compañía bananera de la zona sur $y$ mucho de nosotros y Muchas mujeres nos quedamos sin trabajo ${ }^{34}$.

De esta manera, se constata que en el caso del Frente Amplio, la conversación se esparce hacia temas muy alejados de la elección de Gonzalo Ramírez como presidente legislativo. Al debatir sobre las relaciones del FA con las izquierdas latinoamericanas o sobre el papel de Partido Comunista en la historia de Costa Rica, los comentaristas confrontan visiones políticas radicalmente opuestas. Al igual que los debates sobre el peso de la religión cristiana, estas discusiones sugieren la existencia subyacente de oposiciones intensas y profundas que atraviesan la sociedad costarricense, apoyadas en identidades políticas marcadas. El contenido de las notas, en ambos casos, parece solamente servir como punto de partida para una conversación espontánea en la cual algunos temas se singularizan por su potencial de controversia y por la fuerza que las oposiciones permiten evidenciar.

\footnotetext{
$33 \quad$ Nota $n^{\circ} 1-03 / 05 / 2017$

$34 \quad$ Nota $^{\circ} 1-03 / 05 / 2017$
} 


\section{CONCLUSIÓN}

Los resultados aquí presentados son más bien pistas para la investigación futura, por lo que no se pueden obtener conclusiones definitivas sobre el espacio público en línea a partir del análisis de un poco más de mil comentarios. Con el fin de poner en perspectiva crítica los hallazgos presentados anteriormente, se proponen dos principales direcciones para seguir la exploración de este tema.

Primero, cabe destacar el dinamismo de estos micro-espacios, espacios "terceros" según la definición de Scott Wright, comprobado por la considerable producción en este caso de opiniones sobre asuntos públicos de parte de los seguidores de CRHoy, cuyo volumen demuestra un interés marcado por temáticas supuestamente poco atractivas en la época actual. Muchos comentarios - tal vez su mayoría- son muy cortos, poco argumentados, hasta a veces puras invectivas, pero existe simultáneamente una fracción significativa de comentarios orientados hacia el debate, que contienen argumentos precisos, recordatorios de hechos pasados y que manifiestan la voluntad de producir una interpretación personal del asunto debatido, así como de compartirla o confrontarla con otras.

Se constata en la primera parte del presente artículo que los códigos tradicionales de la expresión pública, tales como la exigencia de una discusión despersonalizada, de alcance general, orientada hacia la definición de un bien común, no han desaparecido en las redes sociales a pesar de los muchos prejuicios que rodean las discusiones en Internet. Sin cederle nada a ningún tipo de entusiasmo ciego en relación con las nuevas tecnologías, pero en lugar de lamentar que el Internet no sea siempre un espacio para discusiones entre personas educadas, parece importante seguir investigando la expresión en línea como espacio de elaboración democrática; no hay otra esfera pública más masiva ni más expresiva.

En segundo lugar, este estudio permite ver de manera llamativa la manera en que las notas de CRHoy tienen un papel de "pretexto" en la discusión. Así como, se observa a lo largo de este artículo, los comentaristas tienen una fuerte propensión a salirse del tema, a ampliar el debate trayendo nuevos elementos para la discusión, hablando de hechos no mencionados por los periodistas del sitio web. Llama la atención la manera en que surgen siempre $y$ casi independientemente del tema tratado por la nota comentada, debates sobre la corrupción política en Costa Rica, la legitimidad de la religión para regular los comportamientos colectivos, incluso hasta el futuro de las izquierdas latinoamericanas.

Esto sugiere la existencia de debates subyacentes en la sociedad costarricense, oposiciones latentes que surgen con fuerza a partir de elementos de la actualidad; en este aspecto, parece que el dinamismo observado a propósito de la elección de Gonzalo Ramírez va mucho más allá del cambio de directorio en la Asamblea Legislativa: esta ocasión institucional $-y$ su contexto particular - sirven como detonante para una expresión polifacética que abarca temas mucho más amplios que los simples hechos comentados. Se cree entonces haber ayudado a comprobar el interés que tiene el estudio de las opiniones en línea, en un modo radicalmente distinto a las encuestas tradicionales: no para cuantificar con precisión las preferencias del público, sino para conectarse con tendencias poco esperadas, gracias a la riqueza de este material espontáneamente producido.

Para terminar, se sugiere una pista para la investigación futura, a partir de la constatación del surgimiento de temas subyacentes de la opinión pública. Consistiría en comprobar de manera amplia y sistemática la autonomía de la agenda política del público en general. De hecho, lo que sugiere el presente estudio es un relativo desfase entre las narrativas mediáticas y políticas tradicionales, $y$ lo que le interesa realmente a los comentaristas de CRHoy; los temas o ángulos valorados por los periodistas, entre otros, el juego parlamentario, las alianzas y traiciones, o la cuestión del gasto público, no son iguales a los que privilegian su público (corrupción, religión, etc.).

Aunque los hechos discutidos sean similares, la manera en que se abordan difiere significativamente ${ }^{35}$. Desde un punto de vista más

35 Este constato concuerda también con varias entrevistas exploratorias realizadas con los 
general, esto confortaría los resultados clásicos de los estudios de recepción sobre la autonomía reflexiva del público (Hoggart, 1971; Morley, 1980), también podría contribuir en matizar la teoría de agenda-setting de McCombs y Shaw (1972): si bien es cierto que en los comentarios analizados, CRHoy construye un marco general para la discusión proporcionando hechos $y$ temas, se observan que algunos surgen con intensidad, independientemente de los contenidos noticiosos.

\section{REFERENCIAS}

Beuscart, J., É. Dagiral, y Parasie, S. (2016). Sociologie d'internet. Paris, Francia: Armand Colin.

Boltanski, L. (2009). De la critique. Paris, Francia: Gallimard.

Boullier, D. (2015). Les sciences sociales face aux traces du big data: opinion, société ou vibrations? Revue Française de Science Politique (65), 805-829.

Bourdieu, P. (1973). L'opinion publique n'existe pas. Les Temps Modernes (318), 12921309.

Cardon, D. (2010). La démocratie Internet. Promesses et limites. Paris, Francia: Seuil.

Desrosières, A. (2008). L'argument statistique. Sciences sociales. Paris, Francia: Presses de l'École des mines.

Farge, A. (1992). Dire et mal dire. L'opinion publique au xvIIE siècle. Paris, Francia: Seuil.

Flichy, Patrice. (2008). Internet et le débat démocratique. Réseaux (150), 159-185.

Gillespie, T. (2014). The Relevance of Algorithms, en Boczkowski P., Gillespie T. y Foot K. A., Media Technologies. Essays on Communication, Materiality and Society (pp.167-193). Boston, EEuu: мIт Press.

protagonistas políticos de esta elección en el marco de este proyecto de investigación, los cuales también parecen valorar temas muy distintos a los que se comentan en Facebook.
Goffman, E. (1982). Interaction Ritual: Essays on Face-to-Face Behavior. New York, Eevu: Pantheon Books.

Greffet, F. y Wojcik, S. (2008). Parler politique en ligne: une revue des travaux français et anglo-saxons. Réseaux (150), 19-50.

Habermas, J. (1999). The Structural Transformation of the Public Sphere: an Inquiry into a Category of Bourgeois Society. Studies in contemporary German social thought. Cambridge, ик: MIT Press.

Hoggart, R. (1971). The Uses of Literacy. Aspects of Workingclass Life, with Special References to Publications and Entertainments. Reprinted. Londres, Inglaterra: Chatto, Windus.

Jones, S. (1995). Cybersociety. ComputerMediated Communication and Community. Thousand Oaks: Sage.

Lemieux, C. (2007). À quoi sert l'analyse des controverses? Mil neuf cent, revue d'histoire intellectuelle (25), 191-212.

McCombs, M. E., y Shaw, D. L. (1972). The Agenda-Setting Function of Mass Media. Public Opinion Quarterly (36),176-187.

Monnoyer-Smith, L. (2006). Citizen's Deliberation on the Internet: An Exploratory Study. International Journal of Electronic Government Research (3), 58-74.

Morley, D. (1980). The Nationwide Audience. Londres, Inglaterra: British Film Inst.

Parasie, S., y Cointet, J. P. (2012). La presse en ligne au service de la démocratie locale. Une analyse morphologique de forums politiques. Revue française de science politique (62), 45-70.

Rheingold, H. (2000). The Virtual Community: Homesteading on the Electronic Frontier. 2nd Revised edition. Cambridge, uк: мाт Press.

Rieder, B. (2013). Studying Facebook via Data Extraction: The Netvizz Application. Proceedings of the 5th Annual ACM Web Science Conference, 346-355. 
Sunstein, C. (2001). Republic.com. Princeton, Eeuv: Princeton University Press.

Wojcieszak, M. (2008). False Consensus Goes Online: Impact of Ideologically Homogeneous Groups on False Consensus. Public Opinion Quarterly, (72), 781-791.
Wright, S. (2012). From 'Third Place' to 'Third Space': Everyday Political Talk in NonPolitical Online Spaces. Javnost-The Public (19), 5-20.

Fecha de ingreso: 24/10/2017 Fecha de aprobación: 04/04/2018 
